\title{
CYP2C19 pharmacogenetics in advanced cancer: compromised function independent of genotype
}

\author{
NA Helsby ${ }^{*, 1,2}$, W-Y Lo', K Sharples ${ }^{3,4}$, G Riley ${ }^{3}$, M Murray $^{3}, K_{\text {Spells }}^{5}$, M Dzhelai ${ }^{6}$, A Simpson ${ }^{6}$ and M Findlay ${ }^{2,3}$ \\ 'Department of Molecular Medicine and Pathology, University of Auckland, Auckland, New Zealand; ${ }^{2}$ Auckland Cancer Society Research Centre, \\ University of Auckland, Auckland, New Zealand; ${ }^{3}$ Cancer Trials New Zealand, University of Auckland, Auckland, New Zealand; ${ }^{4}$ Department of \\ Preventive and Social Medicine, University of Otago, Dunedin, Otago, New Zealand; ${ }^{5}$ Regional Cancer Centre, Auckland District Health Board, Auckland, \\ New Zealand; ${ }^{6}$ Wellington Cancer Centre, Capital and Coast District Health Board, Wellington, New Zealand
}

CYP2C19 is a drug-metabolising enzyme involved in the metabolism of a number of chemotherapeutic agents including cyclophosphamide. Variants of the CYP2CI 9 gene result in a loss of function polymorphism, which affects approximately $3 \%$ of the Caucasian population. These individuals are poor metabolisers (PM) of a wide range of medications including omeprazole (OMP). In healthy subjects PM can be identified through homozygous variant genotype. However, a discordance between CYP2C19 genotype and phenotype has been reported previously in a small study of cancer patients. To investigate whether CYP2CI9 activity was decreased in patients with advanced cancer, CYP2C 19 genotype was determined in 33 advanced cancer patients using PCR-RFLP analysis for the two important allelic variants $(* 2,68 \mathrm{IG}>\mathrm{A}$ and $* 3,636 \mathrm{G}>\mathrm{A}$ ) and the activity of the enzyme was evaluated using the CYP2C 19 probe drug OMP. The activity of the drug-metabolising enzyme CYP2C 19 was severely compromised in advanced cancer patients, resulting in a PM status in $37 \%$ of the patients who had normal genotype. This is significantly $(P<0.0005)$ higher than that would be predicted from the genotypic status of these patients. There was no evidence of a correlation between compromised CYP2CI9 activity and any of the proinflammatory cytokines or acute phase response proteins studied. However, there was preliminary evidence of an association between PM status and low body mass $(P=0.03)$. There is increasing interest in using pharmacogenetics to 'individualise medicine', however, the results of this study indicate that in a cancer population genotyping for CYP2C19 would significantly underestimate the number of phenotypic PM of drugs, such as cyclophosphamide, which may be metabolised by this enzyme.

British Journal of Cancer (2008) 99, I25 I - 1255. doi:I0.1038/sj.bjc.6604699 www.bjcancer.com

(c) 2008 Cancer Research UK

Keywords: loss of function; pharmacogenetics; CYP2C19; advanced cancer; drug metabolism; inflammation

Variation in drug disposition and response is associated with many anticancer agents, in part due to large inter-patient differences in pharmacokinetics (Petros and Evans, 2004). This may be the result of variable expression of hepatic cytochrome P450 (CYP), enzymes which play an important role in the metabolism and elimination of many chemotherapeutic drugs.

CYP2C19 is an important member of the CYP family of enzymes and is involved in the metabolism of a diverse range of drug substrates such as the antiulcer agent omeprazole (OMP) (Desta et al, 2002) and the antimalarial drug proguanil (Ward et al, 1991). Recently, it has become apparent that CYP2C19 is also important in the disposition of a number of chemotherapeutic agents, including cyclophosphamide (Takada et al, 2004; Timm et al, 2005), thalidomide (Ando et al, 2002; Li et al, 2007) and bortezomib (Uttamsingh et al, 2005).

There is a loss of function genetic polymorphism associated with the CYP2C19 enzyme, with the result that individuals who are

\footnotetext{
* Correspondence: Dr NA Helsby, Department of Molecular Medicine and Pathology, University of Auckland, Private Bag 92019. Auckland, New Zealand; E-mail: n.helsby@auckland.ac.nz

Revised 2 September 2008; accepted 8 September 2008
}

homozygous variant (var/var) are 'poor metabolisers' (PM) of drugs that are substrates for CYP2C19 (Desta et al, 2002). Approximately $3 \%$ of Caucasians and up to $30 \%$ of Asians are CYP2C19 PM (Xie et al, 1999). Numerous studies have shown a correlation between variant genotype and PM phenotype in healthy populations (Xie et al, 1999; Desta et al, 2002). Hence, the use of a genotyping test for CYP2C19 metaboliser status is often advocated as a substitute for direct phenotyping with validated probe drugs such as OMP.

However, genetic variability is not the only factor that can affect functional activity of drug-metabolising enzymes; gender, age and co-medications can all play a role in variable CYP expression. In particular, co-medications can mimic the genetic variability observed for CYP2C19 with the result that a patient can become a PM 'phenocopy' due to inhibition (competition) of drug metabolism. However, the importance of chronic disease as a contributor to variable drug disposition is often overlooked.

There is increasing evidence that the activity of CYP is decreased in cancer. In a small study of patients $(n=16)$ with advanced cancer (Williams et al, 2000), all individuals had normal CYP2C19 genotype but $25 \%$ of these patients were CYP2C19 PM phenocopies. However, the mechanism behind this genotypephenotype discordance was not investigated. Previous studies have 
demonstrated that the activity of a different enzyme, CYP3A4, is also decreased in advanced cancer patients and that the decreased activity correlates with inflammatory response (Rivory et al, 2002). An inverse relationship between plasma cytokine (TNF- $\alpha$ and IL-6) concentrations and CYP2C19 activity has been demonstrated in patients with congestive heart failure (Frye et al, 2002). Moreover, CYP2C19 mRNA expression is reported to be downregulated by cytokines in human hepatocyte in vitro cultures (Aitken and Morgan, 2007). Hence, limited evidence indicates that advanced cancer patients may have decreased capacity to metabolise certain chemotherapeutic agents due to decreased CYP activity.

The aim of this study was to confirm whether or not CYP2C19 activity is decreased in patients with advanced cancer and to determine whether a relationship exists between CYP2C19 metaboliser status and inflammatory markers such as cytokines and acute phase response proteins.

\section{MATERIALS AND METHODS}

This study (NTX/05/07/083) was approved by the New Zealand Health and Disability Multi-Regional Ethics Committee. Following full informed written consent, 33 patients ( 18 male, 15 female) with advanced cancer were recruited into the study. All patients were self-reported as Caucasian with the exception of one patient who was Asian. Patients had not received any chemotherapy for at least 4 weeks before the study and were not on any medications that are known inducers or inhibitors of CYP2C19. All patients had adequate hepatic and renal function (creatinine levels $<0.12 \mathrm{mmoll}^{-1}$, AST/ALT $<90 \mathrm{Ul}^{-1}$, ALP $<300 \mathrm{Ul}^{-1}$, bilirubin $\left.<20 \mu \mathrm{moll}^{-1}\right)$.

Blood $(8.5 \mathrm{ml})$ was collected in PAXgene blood tubes and DNA was extracted using the PAXgene ${ }^{\mathrm{TM}}$ blood DNA kit (Qiagen, Hilden, Germany). The CYP2C19 genotype was determined by PCR-RFLP analysis of the two important allelic variants $(C Y P 2 C 19 * 2$ and CYP2C19*3) using previously published methods (Goldstein and Blaisdell, 1996).

The activity of CYP2C19 (phenotype) was determined using OMP as the probe substrate (Balian et al, 1995). The plasma concentration of OMP and the $5^{\prime}$-hydroxy metabolite $\left(5^{\prime}-\mathrm{OH}\right)$ were measured in the patient's plasma $2 \mathrm{~h}$ after oral administration of OMP (20 mg) using previously published methods (Woolf and Matuszewski, 1998; Motevalian et al, 1999; Orlando and Bonato, 2003) with slight alterations. The log OMP hydroxylation index $\left(\log _{10}\left[\mathrm{OMP} / 5^{\prime} \mathrm{OH}\right]\right)$ was used to describe the CYP2C19 metabolic status of the patients. An index of $<1$ has been previously shown to correspond to an extensive metaboliser (EM) phenotype and an index $>1$ is a phenotypic PM (Balian et al, 1995; Kortunay et al, 1997).

Serum cytokine levels (IL- $1 \alpha$, IL- $1 \beta$, IL- 6 , TNF- $\alpha$, TGF- $\beta$ ) were measured by Lincoplex ${ }^{\circledR}$ multiplex immunoassay (LINCO Research, St Charles, MO, USA). The levels of hsCRP were determined by the local medical laboratory testing service using the standard nephelometry assay. The levels of growth hormone and albumin were determined using the commercially available ELISA kits (Growth hormone: R\&D Systems, Minneapolis, MN, USA; Albumin: BioAssay Systems, Hayward, CA, USA).

Statistical analyses were determined using Stata Statistical Software (Release 9, StataCorp LP) and R (R Development Core Team, 2007). Continuous data are reported as median and interquartile range (IQR). Associations between continuous variables were assessed using Spearman's rank correlation, and Mann - Whitney rank-sum tests were used to compare continuous variables across two groups. The discordance between CYP2C19 genotype $v s$ phenotype was assessed using McNemar's test and proportions were compared using Fisher's exact test. Two-sided $P$-values $<0.05$ were considered statistically significant.

\section{RESULTS}

All patients in the study had advanced incurable cancer and a range of primary tumours including breast, lung, gastrointestinal tract, kidney, ovarian and melanoma. Patients were aged between 33 and 79 years, with a mean age of 61.5 years and there were 18 male and 15 female patients in the study.

The CYP2C19 genotype of the patients was determined following PCR-RFLP analysis of the allelic variants $\left(C Y P 2 C 19{ }^{*}\right.$ and CYP2C19*3). Previous studies have shown that individuals who are homozygous variant (var/var) for the CYP2C19 gene have null CYP2C19 activity and are PM. In contrast, individuals with at least one wild-type allele at these loci are classed as EM, that is, $w t / w t$ and $w t / v a r$. One patient was homozygous variant (var/var) for the CYP2C19*3 allele; hence, they are a genotypic PM (gPM) and would be predicted to have null CYP2C19 activity. Genetic PM are more common in Asian populations, and this was the one Asian patient in the study. The CYP $2 C 19{ }^{*} 3$ allele was not observed in any other patient; however, this variant is uncommon in Caucasian populations. The remaining patients were $w t / w t(n=20)$ or $w t / v a r$ $(n=12)$ for the $C Y P 2 C 19{ }^{*}$ allele and hence are all classed as genotypic EMs.

The activity of the CYP2C19 enzyme in the patients was then determined directly using OMP as a probe drug. Two patients had no detectable drug or metabolite in the plasma and were excluded from any further analysis. The medians of the log OMP hydroxylation index were 0.86 and 0.47 for the $w t / v a r$ and $w t / w t$ genotypes, respectively (data shown in Figure 1). The one genotypic PM (var/var) in this study had a log OMP hydroxylation index of 2. This is consistent with the predicted null CYP2C19 activity in this patient. Comparison of the genotype predicted and the measured metabolic status (Table 1) found a statistically significant discordance $(P<0.0005)$. Of the 30 cancer patients with genotypic EM status 11 (37\%) were CYP2C19 PM 'phenocopies'; there was no statistically significant difference between the proportions who were phenotypic PM in the two genotypic EM groups (6 out of 18 for $w t / w t$ and 5 out of 12 for $w t / v a r, P=0.6$ ). Five patients with $w t$ alleles had liver metastases and the hydroxylation index in these patients were $0.11,0.59,1.22,1.32$

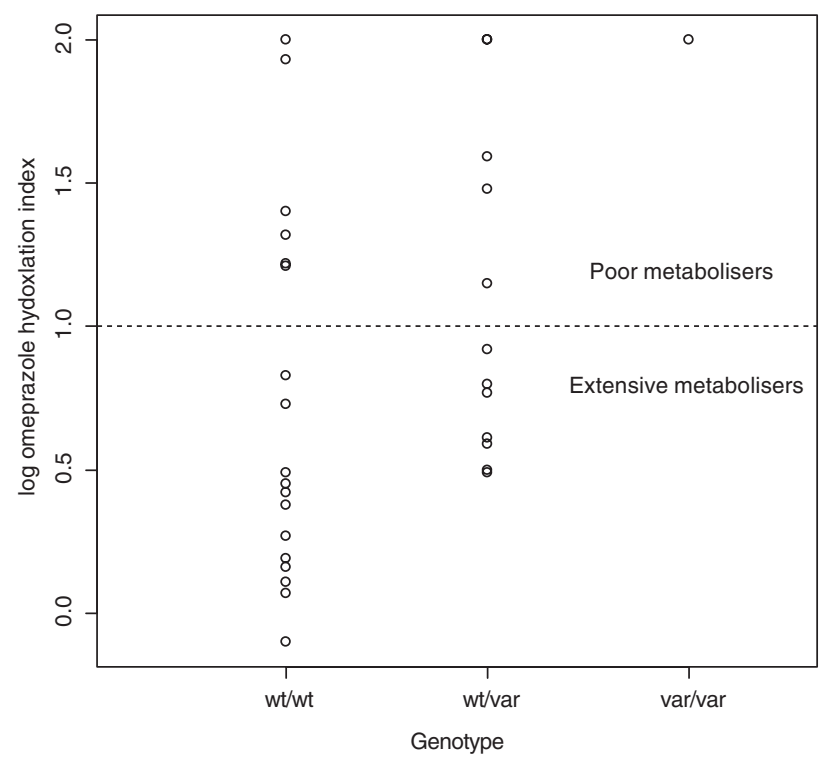

Figure I Distribution of CYP2C19 activity in patients with advanced cancer. Omeprazole (OMP) was used as a probe substrate for the CYP2C19 enzyme and activity was determined using the log OMP hydroxylation index $(\log \mathrm{OMP} \mathrm{HI})$ at $2 \mathrm{~h}$ post-dose. Individuals with a log $\mathrm{OMP} \mathrm{HI}>\mathrm{I}$ are classed as phenotypic poor metabolisers of this drug. 
Table I The predicted CYP2C19 metabolic status (genotype) vs actual CYP2CI9 activity (phenotype) in cancer patients

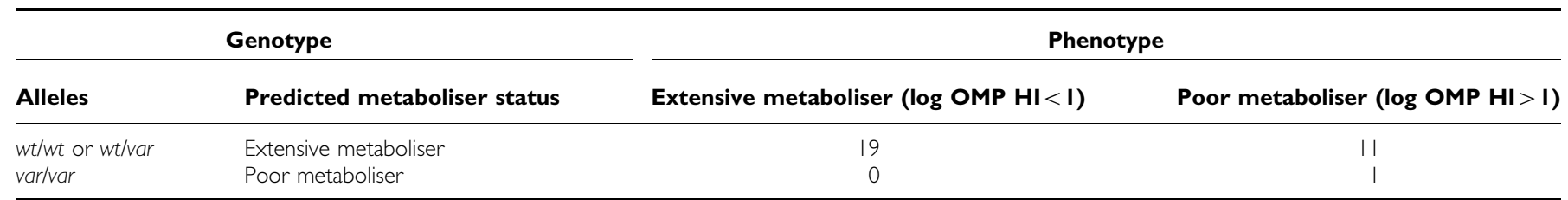

There was a significant discordance between CYP2C19 genotype and phenotype (McNemar's test $P<0.0005)$. Two patients with wt/wt genotype had no detectable drug or metabolite in plasma and hence could not be phenotyped and were not included in this comparison.

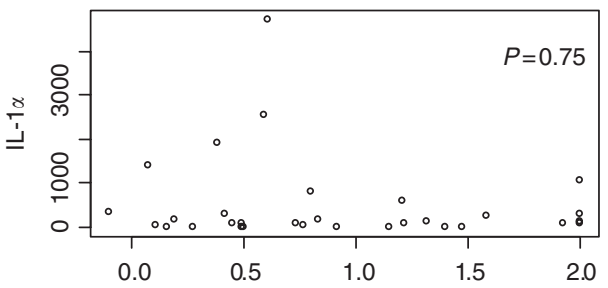

Phenotype (log OMP hydroxylation index)

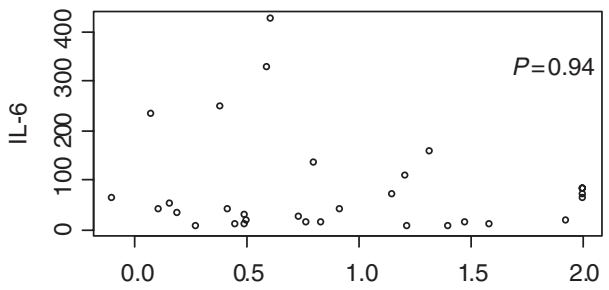

Phenotype (log OMP hydroxylation index)

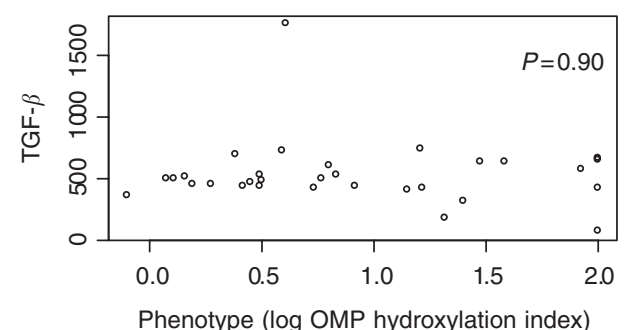

Figure 2 Relationship between CYP2C19 activity and the levels of inflammatory cytokines and CRP. The P-values from Spearman's rank correlation test are shown on the graphs.

and 1.48 , that is, two patients were phenotypic EM and three were phenotypic PM.

A number of studies have demonstrated that acute inflammation can downregulate P450 enzyme expression, (reviewed by Morgan et al, 2008). To determine whether the decreased CYP2C19 activity observed in the cancer patients was related to the inflammatory response, the serum levels of a number of proinflammatory cytokines and the acute phase response protein, CRP were measured. There was considerable variability in the levels of IL- $1 \alpha$, IL- $1 \beta$, IL- 6 , TNF- $\alpha$ and TGF- $\beta$ and these were clearly elevated in some patients, with a median (IQR) for IL- $1 \alpha$ of 107.2 (19.8-408.2); IL-1 $\beta, 1.1$ (0-2.7); IL-6, 40.0 (13.3-89.1); TNF- $\alpha, 6.5$ $(4.0-10.8)$ and TGF- $\beta, 495.0(427.5-627.5) \mathrm{pg} \mathrm{ml}^{-1}$. The acute phase protein, hs-CRP, was above the upper limit of normal $\left(10 \mathrm{mgl}^{-1}\right)$ in 15 patients; however, only five individuals had levels $>40 \mathrm{mgl}^{-1}$. There was no statistically significant correlation between the levels of any individual cytokine and CYP2C19 metabolic activity (log OMP hydroxylation index) (Figure 2, Table 2). Multiple regression analysis also did not reveal any evidence of correlation between inflammatory markers and

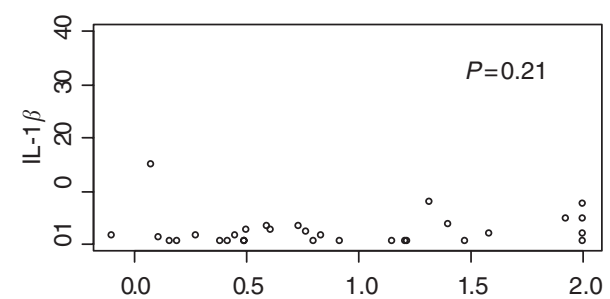

Phenotype (log OMP hydroxylation index)
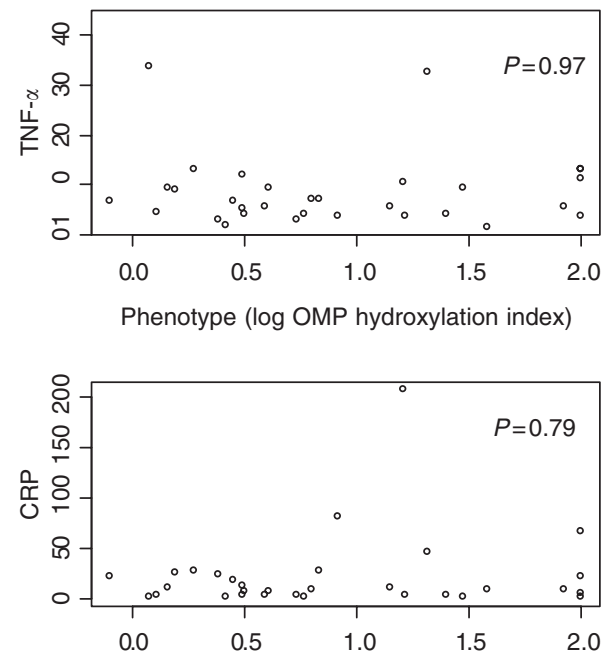

Phenotype (log OMP hydroxylation index)
CYP2C19 activity in the patients. The acute phase inflammatory response also results in hypoalbuminaemia and this has been associated with decreased activity of other CYP enzymes in cancer patients (Rivory et al, 2002). The median albumin concentration in this study was $45.4 \mathrm{gl}^{-1}$ (IQR $43.7-48.8$ ) and only one patient was hypoalbuminaemic (below $35 \mathrm{gl}^{-1}$ ). However, there was no evidence of association between albumin concentrations and $\log$ OMP hydroxylation index in the patients (Table 2).

Owing to the lack of association between proinflammatory cytokines and CYP2C19 activity in the advanced cancer patients, the samples were also analysed for the levels of growth hormone. Administration of growth hormone has been associated with a decrease in OMP hydroxylation index in healthy volunteers (Jürgens et al, 2002). In addition, growth hormone has been reported to be elevated in cancer patients (Dülger et al, 2004). There was considerable variability in the levels of growth hormone in the cancer patients (median $=0.9, \mathrm{IQR}=0.3-3.0 \mu \mathrm{gl}^{-1}$ ); however, all values were within expected daily variation of this hormone and no patients had levels elevated above normal values $\left(>24 \mu \mathrm{gl}^{-1}\right)$. Moreover, there was no evidence of association 
Table 2 Comparison of the phenotypic poor metabolisers and extensive metabolisers

\begin{tabular}{|c|c|c|c|}
\hline & \multicolumn{2}{|c|}{ Phenotype } & \multirow[b]{2}{*}{$P$-value } \\
\hline & $\begin{array}{c}\text { Poor metabolisers }(\text { log OMP HI }>I \text { ) } \\
\text { median (IQ range) } n=12\end{array}$ & $\begin{array}{c}\text { Extensive metabolisers }(\log \text { OMP } \mathrm{HI}<\mathrm{I} \text { ) } \\
\text { median (IQ range) } n=19\end{array}$ & \\
\hline$|L-| \alpha$ & || $1.2(17.7,287.3)$ & I07.2 (20.8, 794.5) & 0.61 \\
\hline $\mathrm{IL}-\mid \beta$ & $1.4(0,39.2)$ & $1.1(0,2.3)$ & 0.35 \\
\hline IL-6 & $65.9(12.0,82.7)$ & $38.0(13.4,134.9)$ & 0.97 \\
\hline TNF & $7.4(3.8,12.5)$ & $6.5(4,9.3)$ & 0.57 \\
\hline Albumin & $46.9(43.4,50.2)$ & $44.8(43.0,48.9)$ & 0.41 \\
\hline Growth hormone & $988.8(222.5,3075)$ & $955.7(496.3,4 \mid 17.2)$ & 0.44 \\
\hline BMI & $27.7(21.7,30.0)$ & $23.7(22.0,27.6)$ & 0.09 \\
\hline
\end{tabular}

Median and interquartile (IQ) ranges are shown for levels of inflammatory cytokines, albumin concentration, growth hormone concentration and body mass index (BMI) aMann-Whitney rank-sum test.

between the levels of growth hormone and CYP2C19 activity (log OMP hydroxylation index) in the cancer patients (Table 2).

Another indicator of disease progression in cancer is cachexia, particularly skeletal muscle loss. Although this study did not directly assess the patients' cachexic status, body mass index (BMI) was determined in these patients at the time of recruitment. There was preliminary evidence of an association between BMI and CYP2C19 activity in the advanced cancer patients; according to the standard cutoff for BMI, among those with low BMI $(<25)$ $62 \%$ (8 of 13 ) were PM compared with $22 \%$ (4 of 18 ) in patients with a high BMI $(>25)(P=0.03)$.

\section{DISCUSSION}

The results of this study indicate that the activity of the drugmetabolising enzyme CYP2C19 was compromised in advanced cancer patients. Of the advanced cancer patients studied who had genotypic EM status, $37 \%$ were PM phenocopies. In healthy subjects, only $3 \%$ of Caucasian subjects are CYP2C19 PM and this is due to the presence of the CYP2C19 loss of function genotype (homozygous variant) in these individuals (Xie et al, 1999).

In a healthy population, the activity of CYP2C19 is bimodally distributed and the antimode of the population (log OMP hydroxylation index $=1.0)$ can delineate between CYP2C19 genotypic EM ( $w t / w t$ and $w t / v a r)$ and genotypic PM (var/var) genotypes. The log OMP hydroxylation index in genotypic EM is reported to be between -0.55 and 0.40 (Balian et al, 1995). In contrast, the activity of the enzyme in the advanced cancer population was not bimodally distributed and the activity of the enzyme was low (log OMP hydroxylation index range -0.10 to 2.0 ) indicating that the overall activity of this enzyme was compromised in this cancer population.

This is not the first study to demonstrate the effect of advanced cancer on drug-metabolising enzymes in advanced cancer. A discordance between CYP2C19 genotype and phenotype has been reported previously in a small study $(n=16)$ of advanced cancer patients (Williams et al, 2000). The higher incidence of phenotypic PM in this study (37\%) compared with an incidence of $25 \%$ (Williams et al, 2000) may reflect small sample variation. However, the high incidence of PM in both studies indicates that decreased CYP2C19 activity in cancer patients is a noteworthy effect that could have clinical significance for chemotherapeutics, such as cyclophosphamide, which may be substrates for CYP2C19.

A number of clinical factors may influence the activity of hepatic enzymes, including co-medications and liver disease. However, no patients in this study had taken any medications that could induce or inhibit CYP2C19 and patients had not received any cancer chemotherapy for at least 4 weeks before the study. Inclusion criteria for the study also required patients to have good hepatic and renal function, in contrast to the previous small study (Williams et al, 2000) in which a number of patients had elevated ALP enzyme and two patients had hepatitis. Although five patients in this study had liver metastases, this did not appear to correlate with compromised CYP2C19 metaboliser status and there was also no relationship with the location of the primary cancer and metaboliser status.

An additional factor hypothesised to affect the activity of hepatic CYP enzymes is inflammation. The activity of another hepatic drug-metabolising enzyme CYP3A is decreased in cancer patients (Rivory et al, 2002) and this decreased activity correlates with the acute phase response status of the patients. In addition, an inverse relationship between CYP2C19 activity and plasma TNF- $\alpha$ or IL-6 concentrations has been reported in patients with congestive heart failure (Frye et al, 2002). A more recent study (Aitken and Morgan, 2007) using cultured human hepatocytes has reported that a number of cytokines can downregulate CYP2C19 mRNA expression in vitro. However, in this study in advanced cancer patients, we could not find evidence of a relationship between CYP2C19 activity and serum levels of cytokines or the acute phase response protein CRP. Nevertheless, alternate cytokines or other inflammatory mediators may be involved in the observed decreased CYP2C19 function in advanced cancer.

The levels of cytokines observed in our study were in the same range as the data reported previously in cancer patients (Rivory et al, 2002) and in patients with congestive heart failure (Frye et al, 2002). But, it should be noted that the levels $\left(\mathrm{pg} \mathrm{m}^{-1}\right.$ ) of the cytokines observed in cancer patients in this study is approximately 100 -fold lower than the $10 \mathrm{ng} \mathrm{ml}^{-1}$ concentrations of IL- 6 and TGF- $\beta$ required to significantly downregulate CYP2C19 mRNA in vitro (Aitken and Morgan, 2007). However, the circulating half-life of cytokines is relatively short and the levels may also be underestimated due to the presence of soluble receptors for these inflammatory mediators. Moreover, little is known about the temporal relationship between circulating cytokine levels and downregulation of CYP in vivo.

Further studies in larger cohorts of patients are required to explore these hypotheses more fully.

We also did not observe a relationship between decreased CYP2C19 activity and the acute phase response protein CRP. This may again be due to the relatively low levels of this inflammatory marker observed in the patients. The relationship between CRP levels and decreased CYP3A activity demonstrated previously (Rivory et al, 2002), occurred at relatively high concentrations of CRP, in contrast only five patients in this study had CRP levels $>40 \mathrm{mgl}^{-1}$. The levels $>40 \mathrm{mg} \mathrm{ml}^{-1}$ are considered to be indicative of acute inflammation (Gill, 2000). Hence, the inflammatory mediators in this population of advanced cancer patients 
may not have achieved levels high enough to have an effect on CYP2C19 activity.

Administration of growth hormone can decrease CYP2C19 activity in man (Jürgens et al, 2002). In addition, growth hormone has been reported to be elevated in cancer patients (Dülger et al, 2004). However, there also did not appear to be a relationship between growth hormone levels and decreased CYP2C19 activity in these patients.

Thus currently, the mediators of the decreased activity of CYP2C19 in advanced cancer are not apparent. However, there was a significant relationship between body mass and CYP2C19 PM status. Although BMI is not a good indicator of cachexia particularly in the late stage disease, as weight loss due to increased muscle and fat catabolism, may be obscured by weight gain due to ascites. Nonetheless, the relationship between low BMI and poor metabolic status is intriguing. Cachexia is associated with increased proinflammatory cytokines particularly IL- 6 and TNF- $\alpha$, increased CRP, decreased albumin production and also decreased GH levels (Dülger et al, 2004) and hence may be a more general biomarker of the inflammatory status in these patients. Alternatively, the overall increased protein catabolism in cachexia may

\section{REFERENCES}

Aitken A, Morgan E (2007) Gene-specific effects of inflammatory cytokines on cytochrome P450 2C, 2B6 and 3A4 mRNA levels in human hepatocytes. Drug Metab Dispos 35(9): 1687-1693

Ando Y, Fuse E, Figg W (2002) Thalidomide metabolism by the CYP2C subfamily. Clin Cancer Res 8(6): $1964-1973$

Balian J, Sukhova N, Harris J, Hewett J, Pickle L, Goldstein J, Woosley R, Flockhart D (1995) The hydroxylation of omeprazole correlates with $S$-mephenytoin metabolism: a population study. Clin Pharmacol Ther 57(6): $662-669$

Desta Z, Zhao X, Shin J, Flockhart D (2002) Clinical significance of the cytochrome P450 2C19 genetic polymorphism. Clin Pharmacokinet 41(12): $913-958$

Dülger H, Alici S, Şekeroğlu M, Erkog R, Özbek H, Noyan T, Yavuz M (2004) Serum levels of leptin and proinflammatory cytokines in patients with gastrointestinal cancer. Int J Clin Pract 58(6): 545-549

Frye R, Schneider V, Frye C, Feldman A (2002) Plasma levels of TNF- $\alpha$ and IL-6 are inversely related to cytochrome P450-dependent drug metabolism in patients with congestive heart failure. J Card Fail 8(5): 315-319

Gill M (2000) A Handbook for the Interpretation Of Laboratory Tests. Diagnostic Medlab Ltd: Auckland

Goldstein J, Blaisdell J (1996) Genetic tests which identify the principal defects in CYP2C19 responsible for the polymorphism in mephenytoin metabolism. Methods Enzymol 272: 210-218

Jürgens G, Lange K, Reuther L, Rasmussen B, Brøsen K, Christensen $\mathrm{H}$ (2002) Effect of growth hormone on hepatic cytochrome P450 activity in healthy elderly men. Clin Pharmacol Ther 71(3): $162-168$

Kortunay S, Basci NE, Bozkurt A, Isimer A, Sayal A, Kayaalp SO (1997) The hydroxylation of omeprazole correlates with $\mathrm{S}$-mephenytoin and proguanil metabolism. Eur J Clin Pharmacol 53(3-4): 261-264

Li Y, Hou J, Jiang H, Wang D, Fu W, Yuan Z, Chen Y, Zhou L (2007) Polymorphisms of CYP2C19 gene are associated with the efficacy of thalidomide based regimens in multiple myeloma. Haematologica 92(9): $1246-1249$

Morgan E, Goralski K, Piquette-Miller M, Renton K, Robertson G, Chaluvadi M, Charles K, Clark SJ, Kacevska M, Liddle C, Richardson T, Sharma R, Sinal C (2008) Regulation of drug-metabolizing enzymes and transporters in infection, inflammation, and cancer. Drug Metab Dispos 36(2): 205-216

Motevalian M, Saeedi G, Keyhanfar F, Tayebi L, Mahmoudian M (1999) Simultaneous determination of omeprazole and its metabolites in human play a more direct role in the compromised CYP2C19 activity. The preliminary finding of a possible association between compromised CYP2C19 activity and low BMI requires further study in a larger cohort of patients.

The implications of compromised CYP2C19 activity are of direct relevance to cancer chemotherapy. The decrease in enzyme activity may significantly impact on the efficacy and toxicity of chemotherapeutic agents that are substrates for this enzyme. Moreover, a pharmacogenomic approach is often advocated to 'personalise' medicine to reduce the incidence of under- and overdosing of patients. However, it is apparent that in cancer patients this approach would considerably underestimate the number of $\mathrm{PM}$ of drugs that are substrates for this enzyme.

\section{ACKNOWLEDGEMENTS}

This study was supported by a research grant from the Cancer Society of New Zealand. W-Y Lo would also like to acknowledge a doctoral scholarship from the Cancer Society of New Zealand. We would also thank the patients for their involvement in this study.

plasma by HPLC using solid-phase extraction. Pharm Pharmacol Commun 5: $265-268$

Orlando R, Bonato P (2003) Simple and efficient method for enantioselective determination of omeprazole in human plasma. J Chromatogr B Analyt Technol Biomed Life Sci 795(2): 227-235

Petros W, Evans W (2004) Pharmacogenomics in cancer therapy: is host genome variability important? Trends Pharmacol Sci 25(9): 457-464

R Development Core Team (2007) R: A Language and Environment for Statistical Computing. R Foundation for Statistical Computing: Vienna, Austria. ISBN 3-900051-07-0, URL: http://www.R-project.org

Rivory L, Slaviero K, Clarke S (2002) Hepatic cytochrome P450 3A drug metabolism is reduced in cancer patients who have an acute-phase response. $\mathrm{Br} J$ Cancer 87: $277-280$

Takada K, Arefayene M, Desta Z, Yarboro CH, Boumpas DT, Balow JE, Flockhart DA, Illei GG (2004) Cytochrome P450 pharmacogenetics as a predictor of toxicity and clinical response to pulse cyclophosphamide in lupus nephritis. Arthritis Rheum 50(7): 2202-2210

Timm R, Kaiser R, Lötsch J, Heider U, Sezer O, Weisz K, Montemurro M, Roots I, Cascorbi I (2005) Association of cyclophosphamide pharmacokinetics to polymorphic cytochrome P450 2C19. Pharmacogenomics J 5: $365-373$

Uttamsingh V, Lu C, Miwa G, Gan L (2005) Relative contributions of the five major human cytochromes $\mathrm{P} 450,1 \mathrm{~A} 2,2 \mathrm{C} 9,2 \mathrm{C} 19,2 \mathrm{D} 6$, and $3 \mathrm{~A} 4$, to the hepatic metabolism of the proteasome inhibitor bortezomib. Drug Metab Dispos 33(11): 1723-1728

Ward S, Helsby N, Skjelbo E, Brosen K, Gram L, Breckenridge A (1991) The activation of the biguanide antimalarial proguanil co-segregates with the mephenytoin oxidation polymorphism - a panel study. Br J Clin Pharmacol 31(6): 689-692

Williams M, Bhargava P, Cherrouk I, Marshall J, Flockhart D, Wainer I (2000) A discordance of the cytochrome P450 2C19 genotype and phenotype in patients with advanced cancer. Br J Clin Pharmacol 49: $485-488$

Woolf E, Matuszewski B (1998) Simultaneous determination of omeprazole and $5^{\prime}$-hydroxyomeprazole in human plasma by liquid chromatographytandem mass spectrometry. J Chromatogr A 828: 229-238

Xie H, Stein C, Kim R, Wilkinson G, Flockhart D, Wood A (1999) Allelic, genotypic and phenotypic distributions of $S$-mephenytoin $4^{\prime}$-hydroxylase (CYP2C19) in healthy Caucasian populations of European descent throughout the world. Pharmacogenetics 9: 539-549 